\title{
Nonthermal graphitization of diamond induced by a femtosecond x-ray laser pulse
}

\author{
N. Medvedev, ${ }^{1, *}$ H. O. Jeschke, ${ }^{2}$ and B. Ziaja ${ }^{1,3}$ \\ ${ }^{1}$ Center for Free-Electron Laser Science, Deutsches Elektronen-Synchrotron DESY, Notkestrasse 85, D-22607 Hamburg, Germany \\ ${ }^{2}$ Institut für Theoretische Physik, Goethe-Universität Frankfurt am Main, Max-von-Laue-Strasse 1, 60438 Frankfurt, Germany \\ ${ }^{3}$ Institute of Nuclear Physics, Polish Academy of Sciences, Radzikowskiego 152, 31-342 Kraków, Poland \\ (Received 27 September 2013; revised manuscript received 25 November 2013; published 20 December 2013)
}

\begin{abstract}
Diamond irradiated with an ultrashort intense laser pulse in the regime of photon energies from soft up to hard $\mathrm{x}$ rays can undergo a phase transition to graphite. This transition is induced by an excitation of electrons from the valence band or from atomic deep shells of the material into its conduction band, which is followed by a transient rapid change of the interatomic potential. Such a nonthermal phase transition occurs on a femtosecond time scale, shortly after or even during the laser pulse. In this work we show that the duration of the graphitization depends on the incoming photon energy: the higher the photon energy is, the longer the secondary electron cascading which promotes the electrons into the conduction band will take. The transient kinetics of the electronic and atomic processes during the graphitization is analyzed in detail. The damage threshold fluence is calculated in the broad photon energy range and is found to be always $\sim 0.7 \mathrm{eV} /$ atom in terms of the average dose absorbed per atom. It is confirmed that the temporal characteristics of a femtosecond laser pulse (at a fixed pulse duration and fluence) do not significantly influence the transient damage kinetics. Finally, the influence of an additional surface layer of high- $Z$ material on the damage within diamond is discussed.
\end{abstract}

DOI: 10.1103/PhysRevB.88.224304

PACS number(s): 41.60.Cr, 64.70.K-, 42.65.Re, 61.80.Ba

\section{INTRODUCTION}

The fourth-generation light sources, the free-electron lasers (FEL), such as FLASH $,{ }^{1}{ }^{\text {LCLS }},{ }^{2}$ SACLA,${ }^{3}$ and FERMI, ${ }^{4}$ stimulate rapid advances in many scientific fields, including investigation of atoms, ${ }^{5,6}$ molecules, ${ }^{7,8}$ clusters, ${ }^{9,10}$ and solids $^{11-13}$ exposed to intense laser fields. It enables creating and probing plasmas, ${ }^{14,15}$ hot dense matter, ${ }^{15-17}$ and warm dense matter, ${ }^{18,19}$ as well as the investigation of the interaction of low-fluence ultrafast laser pulses with matter, with applications to structural studies within solidstate physics, ${ }^{11,20-23}$ nanophysics, ${ }^{24}$ molecular physics, and biophysics. ${ }^{25}$ The presently operating free-electron lasers can produce laser pulses with durations of a few tens down to a few femtoseconds. ${ }^{1-4}$ Combined with recently developed and constantly improving time-sorting pump-probe techniques, they can probe the atomic or electronic dynamics in irradiated materials with a femtosecond resolution. ${ }^{21,22,26}$

These outstanding experimental achievements rely on a good performance of various devices used in FEL experiments. Many of these devices, such as detectors, substrates, x-ray mirrors and diffractive optics, involve carbon-based materials, in particular diamond. ${ }^{27-31}$ Thus, good understanding and accurate modeling of the damage mechanisms within irradiated diamond are crucial for the success of further experiments and the operation of FELs.

Semiconductors under femtosecond irradiation undergo a sequence of processes. ${ }^{11,32,33}$ First, the photoabsorption promotes electrons from the bound states of the valence band or deep atomic shells ( $K$ shell for carbon) to the conduction band. This process occurs during the action of the laser pulse. The deep-shell holes can then decay through Auger processes, which are the dominant relaxation channel for low- $Z$ (light) elements. ${ }^{34}$ The Auger decay gives rise to one more electronic excitation from the valence band to the conduction band, following the relaxation of the $K$-shell hole into the valence band. The released photo- and Auger electrons scatter further via inelastic channels (impact ionization of valence-band or deep-shell electrons of the material) or elastic channels (scattering on atoms or phonons). The impact ionization cascading typically occurs on a femtosecond scale and finishes when the electron energy falls below the impact ionization threshold. ${ }^{35,36}$ In contrast, the elastic phonon scattering can lead to significant electron energy losses only at much longer, typically picosecond, time scales. ${ }^{32,37}$ The exchange of the kinetic energy during interparticle collisions leads to rapid changes of the transient state of the electronic subsystem, which, in turn, induces a change of potential energy. To explain, in covalently bonded materials, the interatomic bonds are strongly dependent on the specific state of the electronic system. Populating a sufficient number of antibonding states in diamond leads to an ultrafast rearrangement of atoms which attempt to minimize the potential energy and a change from dominant $s p^{3}$ to dominant $s p^{2}$ bonding. This triggers the phase transition from diamond to graphite, known as a nonthermal phase transition. ${ }^{38-40}$ Such phase transitions can be extremely fast, on the scale of a (few) hundred femtoseconds. ${ }^{38,39,41}$

To describe the phase transition, we apply the recently developed hybrid model ${ }^{38,42}$ which traces nonequilibrium kinetics of electrons under ultrashort laser irradiation and the following rearrangement of atoms. The predictions of this model were found to be in good agreement with the experimental measurements of the graphitization threshold for diamond at VUV-XUV photon energies. ${ }^{43}$ In the present work, we extend this model to treat higher photon energies, above the $K$-shell threshold. The damage threshold is calculated in a wide range of photon energies up to tens of $\mathrm{keV}$. Our predictions, combined with those from Refs. 38 and 42, cover the whole range of the photon energies available with the currently operational and soon-to-be-commissioned FELs. We also analyze the transient kinetics of electrons and dynamics of atoms following FEL irradiation as a function of the photon energy and of the pulse shape. In particular, the effect of the $K$-shell hole excitation is studied. Additionally, we discuss 
the influence of a layer of high- $Z$ material covering the surface of diamond (200-nm-thick tungsten layer; Ref. 28) on the radiation damage within diamond. We show that the additional extensive secondary electron production within the surface layer leads to an efficient transfer of the radiation energy absorbed therein into the diamond and thus enhances significantly the damage there.

\section{MODEL}

The hybrid model addressing the processes occurring in a semiconductor during its irradiation with VUV rays or $\mathrm{X}$ rays $^{38,42}$ combines four different theoretical approaches described below. The Monte Carlo (MC) method is used to describe photoabsorption and Auger decays of $K$-shell holes, as well as the transient nonequilibrium kinetics of high-energy electrons and their secondary cascading. ${ }^{11,33,35,36,38}$ A temperature equation is applied to describe low-energy electrons, which reach (nearly) thermal equilibrium already during the first few femtoseconds after the beginning of the laser pulse, following the "bump-on-hot-tail" distribution. ${ }^{11,33,44-46}$ The high-energy-electron and the low-energy-electron domains are interconnected, as electrons can gain or lose energy and go from one domain to another. This forms the source/sink terms for the temperature equation, ${ }^{47,48}$ as the changing number and energy of low-energy electrons directly affect their temperature. Additionally, atomic motion and the evolution of the electronic band structure also influence the electron temperature. ${ }^{38}$ The atom dynamics is followed by the classical molecular dynamics simulation method (MD). ${ }^{38-40}$ Finally, the potential energy surface, the collective forces acting on each atom, and the transient electronic band structure are calculated by diagonalizing a tight-binding (TB) Hamiltonian which evolves in time, following the evolution of the atomic configuration. This treatment enables us to trace the modification of the atomic potential caused by the excitations of electrons and, in turn, to address nonthermal phase transitions with our model.

All model details can be found in Ref. 38. Here we only briefly describe the general methodology, focusing on the advances and improvements made upon the previous work. ${ }^{38}$

\section{A. Monte Carlo modeling of photons, high-energy electrons, and $K$-shell holes}

For the modeling of the photoabsorption, the propagation of high-energy electrons, and Auger decays of $K$-shell holes in irradiated diamond, we apply an event-by-event MC scheme that follows trajectories of individual particles. ${ }^{11,33,49-51}$ Incoming photons are absorbed within the simulation box, following the Lambert-Beer law, ${ }^{52}$ with the mean attenuation length taken from Refs. 53-55. The atomic shell which absorbs a photon (valence band or $K$ shell) is chosen randomly among all shells, using their relative photoabsorption cross sections. For the photon energies above the $K$ edge of carbon, the photoabsorption predominantly proceeds through the $K$-shell ionization $(\sim 97 \%)$, while the valence-band ionization is much less probable $(\sim 3 \%){ }^{54}$

The photoelectron energy is determined by the difference between the binding energy of the electron $I_{p}$ and the incoming photon energy $\hbar \omega$. The binding energy of the electron is defined either by the $K$-shell ionization potential $\left(I_{p}=\right.$ $284 \mathrm{eV}^{56}$ ) or by the valence-band level $E_{\mathbf{i}}$ from which this electron is being excited. The valence level is chosen randomly among all the valence levels, which are obtained by the diagonalization of the tight-binding Hamiltonian, $E_{\mathbf{i}}=$ $\left\langle i\left|H\left(\left\{R_{\mathrm{at}}(t)\right\}\right)\right| i\right\rangle$ (see Sec. II C).

Electrons, populating states at energies above $E_{\mathrm{CB}}+E_{\text {cut }}$, where $E_{\mathrm{CB}}$ represents the conduction-band minimum and the cutoff energy is $E_{\text {cut }} \approx 10 \mathrm{eV}$, are then treated as individual particles within the $\mathrm{MC}$ routine ( $E_{\text {gap }} \approx 5 \mathrm{eV}$ for diamond). From now on we will refer to them as "high-energy electrons." Electrons with lower energies are attributed to the "temperature" domain (see Sec. II B).

This specific value of the cutoff energy has been chosen to include the exponential tail of the low-energy Fermi distribution" $11,33,44-46$ in the "temperature" domain. In order to prove that our results are independent of a particular choice of $E_{\text {cut }}$, we performed test calculations varying the value of $E_{\text {cut }}$ by a few $\mathrm{eV}$ around the cutoff value of $10 \mathrm{eV}$. This variation did not affect the calculation results. This value also corresponds to the top of the conduction-band states described by the tight-binding method (Sec. II C) in our model.

Each high-energy electron can further scatter on the $K$ shell electrons if its energy is higher than the binding energy of the $K$ shell, $E_{e}>I_{p}$; otherwise, only the scattering on the valence-band electrons is possible. We neglect the elastic scattering of electrons on phonons since, as discussed above, this interaction contributes only on much longer time scales than those considered here.

Scattering of a high-energy electron is treated with the complex dielectric function (CDF) formalism ${ }^{38,51,57,58}$ : the cross sections $\sigma_{i}\left(E_{e}\right)$ for electron scattering within solid diamond are obtained from optical data, ${ }^{53,54}$ extended for the case of finite momentum transfer $\hbar q$. The cross section is then calculated from the complex dielectric function $\epsilon\left(\omega_{e}, q\right)$ as

$$
\frac{d \sigma_{i}\left(E_{e}, \hbar \omega_{e}\right)}{d \hbar \omega_{e}}=\frac{2 e^{2} n_{e}}{\pi \hbar^{2} v^{2}} \int_{q_{-}}^{q_{+}} \frac{d q}{q} \operatorname{Im}\left(\frac{-1}{\epsilon\left(\omega_{e}, q\right)}\right),
$$

with $q_{ \pm}=\sqrt{2 m_{e} / \hbar^{2}}\left(\sqrt{E_{e}} \pm \sqrt{E_{e}-\hbar \omega_{e}}\right)$. Here the cross section also depends on the energy $\hbar \omega_{e}$ gained by an electron in a collision and is integrated over the transferred momentum $q, e$ denotes the electron charge, $n_{e}$ is the transient electron density of the low-energy domain, $\hbar$ is the Planck constant, and $v$ is the incident electron velocity corresponding to the energy $E_{e}$.

The coefficients for the parametrization of $\epsilon\left(\omega_{e}, q\right)$, written as a set of Drude-like functions, ${ }^{51,57}$ are shown in Refs. 38 and 42 . As the band structure of the material is changing during the irradiation, one can expect that the CDF and thus the cross section for electron scattering will also evolve in time. This effect might be significant at high-fluence irradiation, leading, e.g., to plasma creation. ${ }^{44,59}$ However, as we have recently demonstrated in Ref. 42, the effect is small for the lowfluence case leading to the structural transition of diamond, as considered here. Moreover, as will be shown in Sec. III, the electron cascading finishes before the phase transition to graphite occurs, and thus, the electrons are interacting with almost undisturbed diamond. Therefore, we neglect the 
corresponding minor changes in the electron-scattering cross sections.

With our model we trace only the energy dependence of electrons, applying periodic boundary conditions in real space. The spatial propagation is disregarded. This approximation is valid as long as the electron diffusion and heat diffusion do not contribute significantly to the overall electron kinetics. This is the case considered here: on subpicosecond time scales following an x-ray irradiation, which homogeneously heats up the sample down to a few micrometers depth within the laser spot of a few microns in size. We also exclude from our considerations the thin near-surface layer, from which the high-energy electrons could escape outside of the material, as discussed in Ref. 38. As the electron mean free path is significantly shorter than the photon attenuation length, it justifies the application of periodic boundaries in the bulk.

When a high-energy electron collides with the valence-band or deep-shell electrons, the probabilities for these collisions are estimated with the appropriate scattering cross sections. Electron energy loss in a collision is calculated with the differential cross section. ${ }^{38,49}$ The electron energy is then reduced by the energy $\Delta E$, transferred in the collision to a secondary electron. The initial energy of the secondary electron is equal to the difference between the transferred energy and the binding energy of the level, from which this electron has been ionized.

If the final energy of the incident electron falls below $E_{\text {cut }}$, this electron is removed from the MC domain and added to the temperature domain. The same energy check is performed for the secondary electron.

After photoionization or impact ionization of the $K$ shell, a hole is left behind. This hole will decay via Auger processes which are predominant for carbon. ${ }^{34}$ We use the Poissonian distribution to model the Auger decay with an average decay time of 8 fs. $^{34}$ When the $K$-shell hole relaxes during an Auger process, one electron is additionally promoted from the valence band into high-energy states of the conduction band, leaving another hole in the valence band. The energy level of the valence band, from which the Auger electron was taken, is chosen randomly. The Auger electron receives the excess energy and is then treated exactly as other secondary electrons. The holes in the valence band and their energies contribute to the source term of the temperature equation.

The MC tracing of photons, electrons, and $K$-shell holes is split into time steps, which are merged with the temperature equation and molecular dynamics time steps. Each time step of MC is iterated more than $10^{4}$ times. The calculated electron distributions are ultimately statistically averaged.

\section{B. Temperature equation for low-energy electrons}

Low-energy electrons and their energy, resulting from MC calculations, are added to the total number of electrons in the valence band and in the low-energy part of the conduction band. ${ }^{38,47,48}$ Assuming a Fermi distribution for these electrons, one can determine their corresponding chemical potential and temperature. ${ }^{38}$ The evolution of these two quantities determines the evolution of the electron distribution and contributes to the potential energy surface (see Sec. II C).
In addition to the energy exchange between high- and low-energy electron domains through scattering processes, the atomic motion also affects the electron distribution. These additional contributions to the electron distribution originate from the evolving electronic band structure and the energy conservation imposed upon the particles within the simulation box. The latter condition implies that the energy lost or gained by the atoms during their motion is transferred to the low-energy electrons, i.e., added to the source term of the temperature equation.

As will be shown in Sec. III, the density of the excited electrons reached after FEL irradiation with fluences considered here is of the order of $10^{21} \mathrm{~cm}^{-3}$. This is a very high density for an electron plasma. Its (partial) thermalization is then known to be very rapid. ${ }^{11,33,44-46}$ However, this electron density is only a fraction of a percent of the solid density. It then does not invalidate the applicability of the ground-state-based approach to the band structure of the solid which is presented in the next section.

\section{Tight-binding molecular dynamics to follow atomic system}

Atomic dynamics is followed by the MD technique. ${ }^{38-40}$ We apply the Parrinello-Rahman method, which allows the simulation box to change its size and shape in time while keeping the pressure or the volume constant. ${ }^{60}$ As we have shown recently, the constant pressure vs constant volume simulations yield very similar results for the femtosecond-long irradiation of diamond. ${ }^{42}$ The Parrinello-Rahman method introduces additional equations of motion for the vectors spanning the simulation box, which depend on the ambient pressure. Enabling the simulation box to change its volume implies changing the density of the material, which, after the phase transition, can then adjust to a new equilibrium value.

In the MD simulations we use the velocity Verlet algorithm $^{38,39}$ with a time step of 0.1 fs. Our simulation box contains 216 atoms with periodic boundary conditions. The atomic system relaxes from an initial configuration for a few hundred time steps prior to the start of the laser exposure.

The potential energy surface is calculated as in Refs. 39 and 40 :

$$
\Phi\left(\left\{r_{i j}(t)\right\}, t\right)=\sum_{\mathrm{i}} f_{e}\left(E_{\mathrm{i}}, t\right) E_{\mathrm{i}}+E_{\mathrm{rep}}\left(\left\{r_{i j}\right\}\right) .
$$

The transient electron distribution function $f_{e}\left(E_{\mathrm{i}}, t\right)$, which is the Fermi function obtained with the temperature equation, as described in Sec. II B, enters Eq. (2). Thus, the time-dependent electronic distribution affects the atomic motion. A possible slight deviation of the exact distribution function from the equilibrium Fermi shape affects only negligibly the atomic motion and the graphitization process. ${ }^{61}$ Additionally, the transient energy levels $E_{\mathrm{i}}$ and the core-core repulsion terms $E_{\text {rep }}\left(\left\{r_{i j}\right\}\right)$, depending on the distance between each pair of atoms $i$ and $j$, enter Eq. (2). The details of these terms can be found in Ref. 38. The forces acting between atoms are calculated with help of the Hellman-Feynman theorem using Eq. (2). ${ }^{39,40}$

The Hamiltonian $H\left(\left\{R_{\mathrm{at}}(t)\right\}\right)$, applied to calculate electronic energy levels, is written in terms of the transferable tight-binding method, depending on the transient positions 
$R_{\mathrm{at}}(t)$ of all the atoms in the simulation box. ${ }^{38,39,62}$ The tight-binding coefficients were adjusted in Ref. 62 to reproduce many different atomic configurations of carbon, such as diamond, graphite, a cubic lattice, and a linear chain of carbon atoms, up to a carbon dimer. Thus, this method is fully capable of tracing phase transitions induced in diamond.

We assumed, however, that an excitation of a high-energy electron, as well as a creation of a $K$-shell hole, does not change the tight-binding coefficients. This assumption is justified for low photon fluences when the total number of created $K$-shell holes and of high-energy electrons released is small when compared to the total number of electrons within the valence band. This assumption holds here since we simulate the low-fluence irradiation of diamond around its graphitization threshold. This graphitization threshold corresponds to the excitation of only a small fraction of electrons and holes, as will be shown in the next section.

\section{RESULTS}

\section{A. Damage threshold in diamond}

We calculated the damage threshold of diamond after its irradiation with pulses of different photon energies up to a few tens of $\mathrm{keV}$. The damage threshold fluence, corresponding to the fluence above which diamond turns into graphite, was estimated in a series of simulations with different pulse fluences at a fixed photon energy. For all simulations performed, a Gaussian laser pulse with a FWHM of 10 fs was used.

The calculations were performed at various incoming photon energies. The VUV-XUV range was studied in our previous work. ${ }^{43}$ It was found that in this regime the critical fluence for the material damage was $E_{\mathrm{th}}=0.7 \mathrm{eV} /$ atom in terms of the absorbed dose, in good agreement with the experimental findings. ${ }^{43}$ For photon energies above the $K$ edge, our simulations indicate that, although the transient kinetics is different as it includes $K$-shell hole creation and relaxation (for details see Secs. III B and IIIC), the damage threshold fluence remains the same. The value of the threshold fluence for the graphitization of diamond then seems to be universal and largely independent of the photon wavelength. Recently available experimental results for amorphous carbon, recorded for 7 - and $12-\mathrm{keV}$ photon energies, ${ }^{27}$ indirectly support this conclusion.

After translating the dose units into the units of the incoming fluence (using the photon attenuation length for this conversion ${ }^{53,54}$ ), we obtain Fig. 1, which shows the dependence of the damage threshold fluence on the photon energy. Figure 1 can be used to evaluate the radiation tolerance of optical elements of FELs and thus to estimate the pulse parameters for future experiments with free-electron lasers in a wide range of photon energies.

However, at this point we should recall that we applied periodic boundary conditions in our model neglecting the heat transport from the simulation box. This approximation is justified for the case when the heat diffusion plays a minor role, i.e., when it acts on time scales much longer than the phase transition (>100 fs). It is indeed the case for most of the considered photon energies, apart from the region where

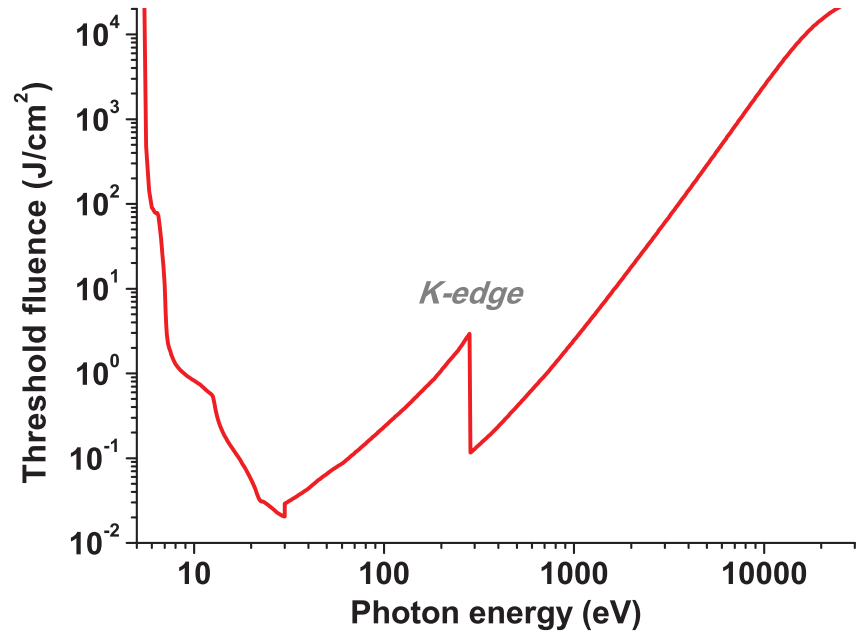

FIG. 1. (Color online) Estimated damage threshold fluence for the graphitization of diamond as a function of photon energy.

the photon attenuation length follows the plasmon minimum, reflected in the minimum of the damage threshold (region of $\sim 15-60 \mathrm{eV}$ ). At these photon energies, large gradients of temperature might occur due to the small skin depth, i.e., photon attenuation length. They can trigger strong diffusion effects on time scales comparable with the phase transition time scale, which would increase the damage threshold.

\section{B. $K$-shell excitation}

To analyze the effect of the $K$-shell excitation on the electron and atom dynamics, we performed calculations for the photon energies around the $K$ edge at 280 and $300 \mathrm{eV}$. The first considered energy is slightly below the $K$ edge, while the second one lies above it. In both cases we used the absorbed dose of $0.85 \mathrm{eV} /$ atom, adjusting the incoming fluence. This dose was chosen to be slightly above the damage threshold in order to enable a fast phase transition but not too high to ensure that no further damage to the material (no ablation or plasma formation) could occur. In what follows we will differentiate between the energy of electrons, the energy of $K$-shell holes, and the energy of atoms. The latter excludes the energy of $K$-shell holes.

After irradiation with a laser pulse of $300-\mathrm{eV}$ photon energy, part of the pulse energy is transiently stored in the $K$-shell holes, while for the case below the $K$ edge (photon energy of $280 \mathrm{eV}$ ), the total energy is distributed only among the electrons and atoms (see Fig. 2). Since $K$-shell holes are absent in the $280-\mathrm{eV}$ irradiation case, the energy of the atoms and electrons then coincides with the total energy plotted in the top panel of Fig. 2.

In the case of the photon energy above the $K$ edge, the $K$-shell holes quickly release their energy back to the electron subsystem via Auger decays. Afterwards, the electronic and atomic dynamics proceed in the same way for the cases below and above the $K$ edge. The strong decrease of the potential energy of atoms corresponds to the increase of their kinetic energy. It occurs during the nonthermal phase transition, as was described in Ref. 38. Thus, one can conclude that the phase transition occurs on similar time scales for the cases 


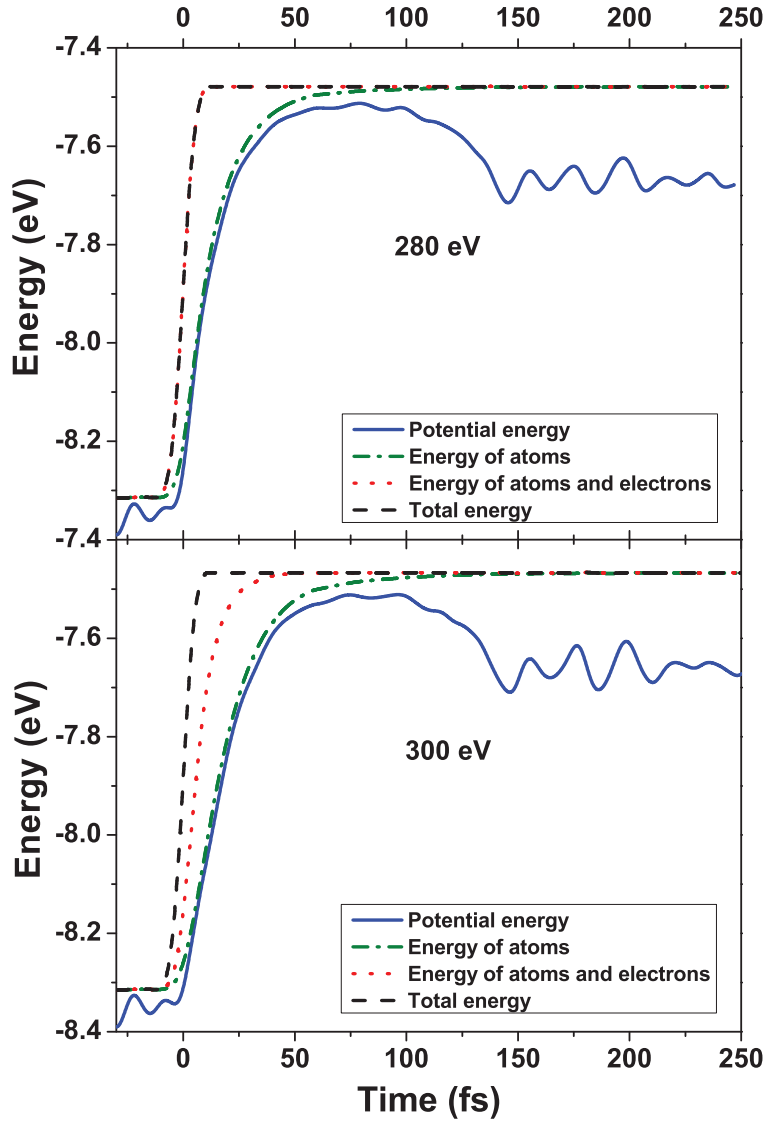

FIG. 2. (Color online) Redistribution of energy among atoms, electrons, and $K$-shell holes after a 10-fs FWHM laser pulse, providing the average absorbed dose of $0.85 \mathrm{eV}$ per atom. (top) The $280-\mathrm{eV}$ photon energy, below the $K$ edge; (bottom) $300-\mathrm{eV}$ photon energy, above the $K$ edge. The black dashed curve is the total energy of the system, the red dotted line is the sum of the energies of the atoms and electrons (the total energy excluding the energy of $K$-shell holes), the green dash-dotted line is the energy of the atoms, and the blue solid line is the potential energy of the atoms.

below and above the $K$ edge, with almost no delay caused by the $K$-shell excitation and Auger decays. The negligible delay of the electron kinetics and of the energy restoration does not affect the damage threshold in terms of the absorbed dose. However, due to the discontinuity of the photon attenuation length at the $K$ edge, one can observe a drop in the damage threshold fluence, as shown in Fig. 1. The delay in the energy release and thus in the kinetics of the phase transition might not be observable in experiments; however, the difference in the photon attenuation lengths affects the depth of the damaged region in the sample. This should be experimentally resolvable.

Within our model, the excitation of the $K$ shell leads only to a transient storage of the energy in a $K$-shell hole, which is then released via an Auger decay. Our treatment neglects a possible perturbation of the local band structure by the hole or recoil effects by the hole relaxation. Such effects can lead to the formation of point defects in diamond. ${ }^{63}$ However, in the low-fluence regime, as used in our simulations, we can neglect these effects since they would appear for no more than $0.05 \%$ of atoms (and at high photon energies even more

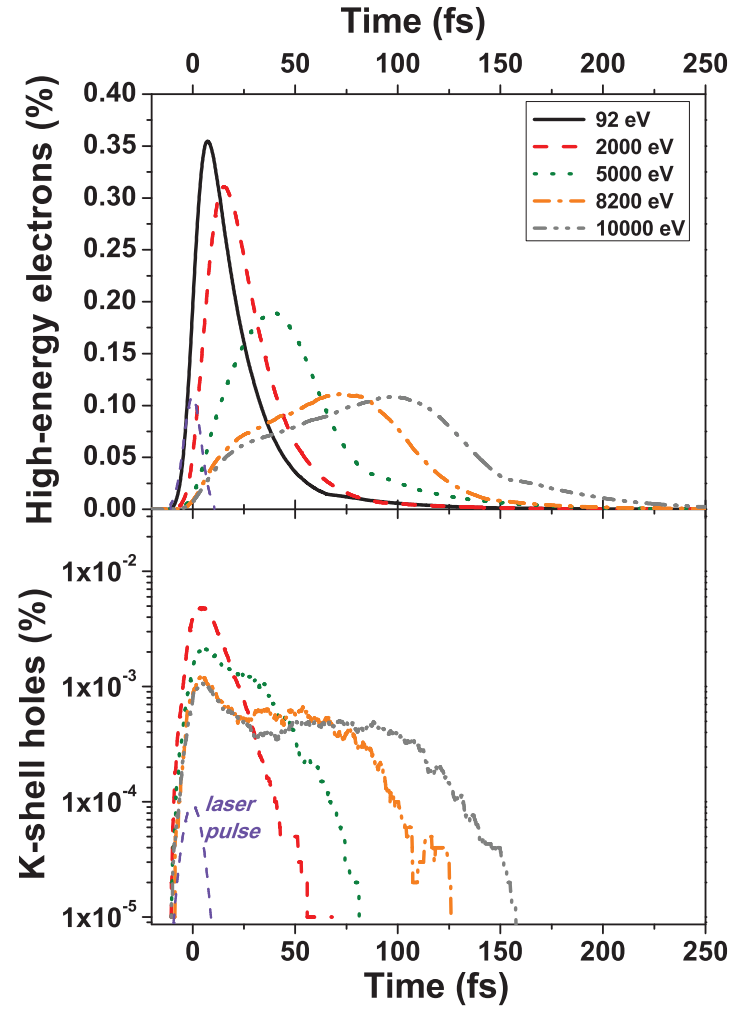

FIG. 3. (Color online) (top) Fraction of high-energy electrons (with energy above $E_{\text {cut }}$, belonging to the MC domain) generated at different photon energies. (bottom) Fraction of $K$-shell holes generated at different photon energies (note the logarithmic scale). Both are normalized to the initial number of valence-band electrons. The laser-pulse profile is schematically shown as a dashed violet line.

rarely; see Fig. 3). The effect of such rare events could become visible only after accumulation of defects, ${ }^{64}$ e.g., in multishot experiments.

\section{Effect of incoming photon energy on transient kinetics}

The higher the incoming photon energy is, the more energy the primarily ionized electrons receive. These electrons perform secondary ionizations, losing part of their energies in collisions. ${ }^{33}$ Therefore, the higher the initial energy of the photoelectron is, the more collisions the photoelectron performs until it loses its energy below $E_{\text {cut }}{ }^{65}$ Figure 3 shows the fraction of high-energy electrons (top panel) and of $K$-shell holes (bottom panel) as functions of time for different photon energies. For all cases the same laser-pulse shape was used: a Gaussian temporal envelope with 10-fs FWHM, delivering the absorbed dose of $0.85 \mathrm{eV}$ per atom.

The secondary electron cascading predominantly proceeds with valence-band ionizations; however, with a probability of $<1.5 \%$ it can also involve $K$-shell impact ionizations. The $K$-shell holes can be produced in the secondary process if the photon energies lie above $2 I_{p}$, as only in this case do photoelectrons gain sufficient energy to perform secondary $K$ shell ionizations. If the photon energy allows photoelectrons to perform secondary impact ionizations in the $K$ shell, the $K$-shell holes are produced during and also after the laser pulse (see Fig. 3). Since we fixed the value of the absorbed 


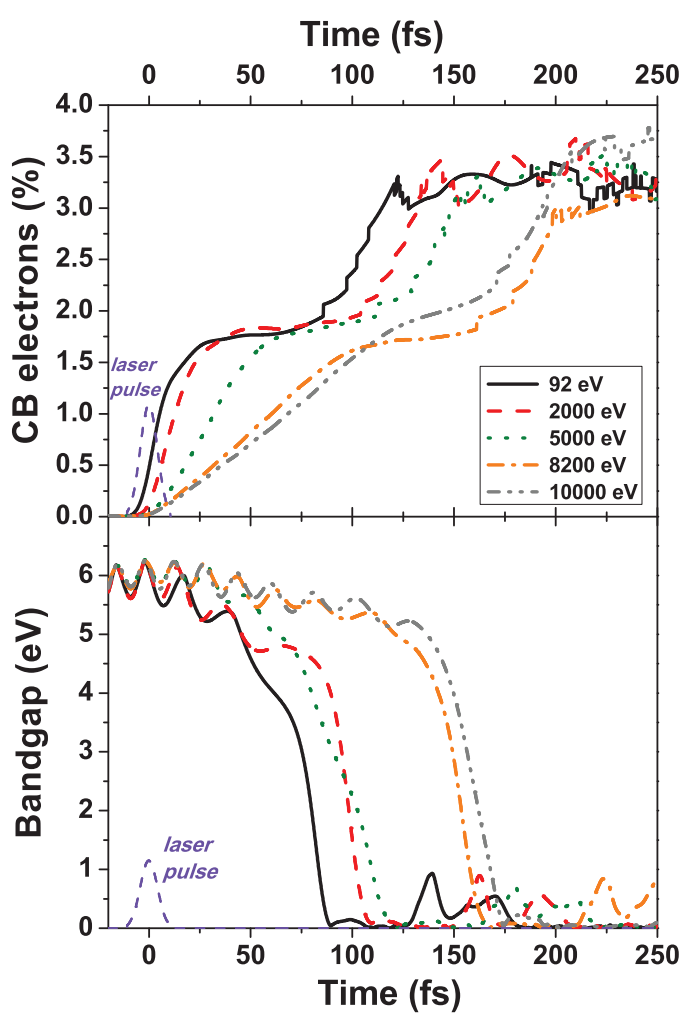

FIG. 4. (Color online) (top) Fraction of conduction-band electrons normalized to the initial number of valence-band electrons for different photon energies. (bottom) Band gap of the irradiated diamond. The laser-pulse shape is schematically shown as a dashed violet line.

dose $D_{\mathrm{ph}}$ for all photon energies, the number of absorbed photons $N_{\text {ph }}$ decreases with the increase of the photon energy: $D_{\mathrm{ph}}=N_{\mathrm{ph}} \hbar \omega=$ const. Such very low densities of $K$-shell holes and high-energy electrons confirm our assumption about their negligible influence on the overall kinetics within the irradiated diamond.

High-energy electrons primarily perform secondary impact ionizations of the valence-band electrons, promoting them into the conduction band. After losing the energy below $E_{\text {cut }}$, high-energy electrons eventually fall into the Fermi sea of the valence- and conduction-band electrons. This increases the total energy and temperature of low-energy electrons and the number of conduction-band electrons (Fig. 4). The top panel of Fig. 4 shows the increase in the number of conduction-band electrons observed at the same laser-pulse parameters as in Fig. 3. The evolution of the free-electron density proceeds in two steps: ${ }^{43}$ first, the fraction of free electrons increases up to $\sim 1.6 \%$ of the initial number of valence electrons due to photoionization and impact ionization processes. The plots show clearly that the higher the photon energy is, the longer the secondary cascading takes. For the case of $10-\mathrm{keV}$ photons, the cascading lasts up to 100 fs longer than the cascading in the case of $92-\mathrm{eV}$ photon energy. At the end of the cascading, the same conduction electron density is reached in both cases.

The second stage starts a few tens of femtoseconds later. It further increases the fraction of free electrons up to $\sim 3.5 \%$.
This increase is due to the collapse of the band gap, which promotes more electrons from the valence to the conduction band (see the bottom panel of Fig. 4). As already shown in Ref. 43, the band gap starts to collapse when the fraction of the conduction-band electrons reaches a value of $\sim 1.5 \%$ of the initial number of valence electrons, which corresponds to an absorbed dose of $\sim 0.7 \mathrm{eV} /$ atom. The induced collapse of the band gap and the increase of the free-electron density trigger a fast relocation of atoms to their new equilibrium positions in the graphite state. ${ }^{43}$ In all calculations, only the graphite with $A B$-layer stacking was observed, not $A A$ or $A B C$ stacking. ${ }^{66,67}$

At the end of the nonthermal phase transition, the material appears to be "swollen." It tries to reach the normal graphite density on longer time scales. Such density relaxation has been observed in the simulations. As under the experimental conditions the laser spot is partly constrained by the unirradiated surrounding lattice, the finally formed graphite was found in experiments to be overdense. ${ }^{43}$

The delay in the electron density increase, caused by the long-lasting cascading for high photon energies, delays the nonthermal graphitization up to a hundred femtoseconds. This can be seen in Fig. 5. The delay is indicated by the sharp decrease of the potential energy of the atoms, which shifts from $\sim 140$ fs for $92-\mathrm{eV}$ photon energy up to more than 240 fs for $10-\mathrm{keV}$ photon irradiation. Such time scales can be resolved in experiment with modern pump-probe techniques. ${ }^{21,22}$ Both x-ray-pump-X-ray-probe schemes, ${ }^{23}$ measuring the atomic positions directly, and $\mathrm{x}$-raypump-visible-light-probe schemes, ${ }^{21,22}$ accessing the data on the electronic structure and the density of the conduction-band electrons, could be employed for this purpose. Time-resolved measurements of the graphitization process for different photon energies could confirm our model results and would be an opportunity to learn more about the nature of the nonthermal phase transition.

One can also observe that the contribution of $K$-shell holes to the overall sample kinetics decreases with the increase of the photon energy: the atomic plus electronic energy almost coincides with the total energy of the system for photon energies of $5 \mathrm{keV}$ and higher. That is because the ratio of the energy stored in $K$-shell holes (sum of their ionization potentials) to the energy of emitted electrons decreases with the increase of the photon energy. Less and less energy is then stored in holes compared to the energy of emitted photoelectrons.

\section{Pulse shape}

All simulations performed up to now in this paper assumed a Gaussian temporal profile of the laser pulse. It is, however, known that the modern FELs are operating in the self-amplified stimulated emission (SASE) mode, ${ }^{68,69}$ which provides a temporally incoherent, spiky structure of the laser-pulse envelope. The question might arise as to how much the specific temporal profile of the laser pulse affects the results. In the linear optics regime ${ }^{70}$ which is considered here, this effect should be small. ${ }^{45}$ To confirm this, we performed a series of simulations for three different laser-pulse profiles: Gaussian, flat top, and a spiky pulse attempting to mimic a typical SASE 


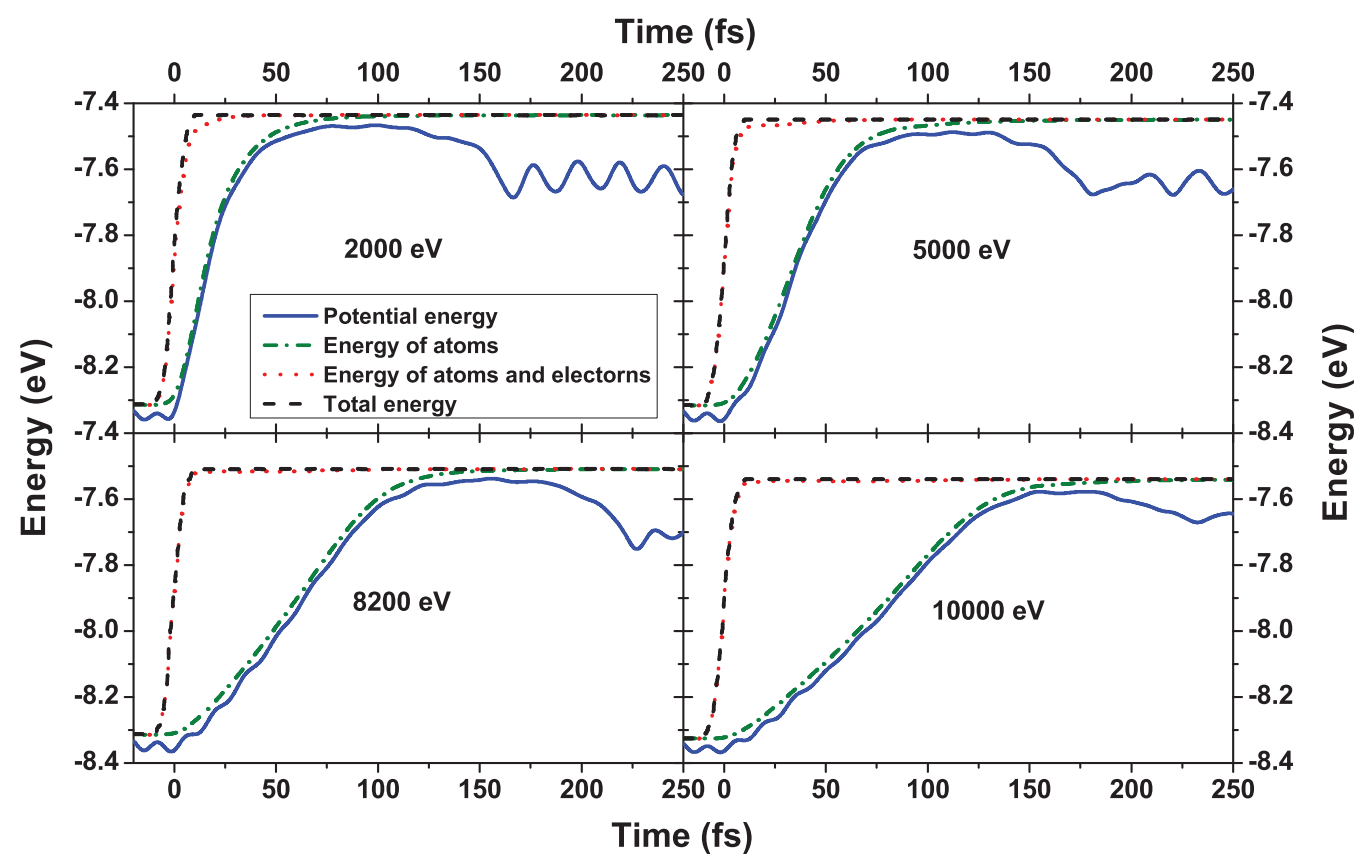

FIG. 5. (Color online) Redistribution of energy between atoms, electrons, and holes during and after the 10-fs FWHM (delivering an average absorbed dose of $0.85 \mathrm{eV}$ per atom) at different photon energies: $2,5,8.2$, and $10 \mathrm{keV}$. In all panels the black dash-dotted curve is the total energy of the system, the red dotted line is the sum of the energies of atoms and electrons (excluding the energy of $K$-shell holes), the green dashed line is the energy of the atoms, and the blue solid line is the potential energy of the atoms.

profile while keeping the duration and the fluence of all pulses fixed.

The results confirm that the pulse shape has no influence on the damage threshold because for femtosecond pulses the threshold depends only on the total deposited dose. The transient kinetics is also almost independent of the temporal laser-pulse shape. Figure 6 shows a comparison of the three pulses, each of which had a photon energy of $300 \mathrm{eV}$, a duration of $10 \mathrm{fs}$, and an absorbed dose of $0.85 \mathrm{eV} /$ atom. Only during the laser pulse did the slopes of the increase of the total energy in the system under irradiation slightly differ, reflecting the specific temporal structure of the pulse. These differences are minor and are totally smoothed out already by the end of the pulse. Other time-dependent observables, such as electron density, $K$-shell density, and band gap, are affected even less. After the end of the pulse, the relaxation kinetics proceeds similarly in all three cases. Thus, we can conclude that during a femtosecond laser irradiation, the specific pulse envelope is of minor importance, and the material excitation and relaxation are defined by the total deposited dose, the pulse duration, and the incoming photon energy.

\section{DISCUSSION}

We have estimated the damage threshold of diamond for a wide range of photon energies. It turns out to be equal to $\sim 0.7 \mathrm{eV} /$ atom in terms of the absorbed dose and is almost independent of the photon wavelength. In terms of the incoming fluence, the threshold translates to the threshold fluence shown in Fig. 1.

In a recent experimental work Uhlén et al. ${ }^{28}$ measured the damage threshold of diamond covered with a 200-nm-thick layer of tungsten. This multilayer sample was irradiated with an $8.2-\mathrm{keV}$ photon laser pulse. The measured damage threshold of this structure appeared to lie between 59 and $99 \mathrm{~J} / \mathrm{cm}^{2}$, which in terms of the absorbed dose would yield $0.027-0.046 \mathrm{eV}$ per atom. The authors suggest that the initial tungsten layer may have had an effect on the damage in diamond. However, it was not clear how strong this effect could be, particularly considering that tungsten evaporates very fast under those irradiation conditions. ${ }^{28}$

The comparison of these results with our calculated damage threshold suggests that the effect is large. The damage threshold is reduced from 0.7 down to $0.027 \mathrm{eV} /$ atom by the presence of the tungsten layer. This can be understood as follows. For 8.2-keV photon energy, the photoabsorption in tungsten is over 200 times more efficient than in diamond. ${ }^{54}$ Photons of $8.2 \mathrm{keV}$ are predominantly absorbed by the $3 \mathrm{~s}$ state of tungsten, which has a binding energy of $2820 \mathrm{eV} .^{56}$ The next state, $2 s$, is too tightly bound to contribute to the photoabsorption $\left[I_{p}(W, 2 s)=10207 \mathrm{eV}\right] .{ }^{56}$ Thus, the photoabsorption in tungsten produces electrons with an energy of $E_{e}=\hbar \omega-I_{p}=5380 \mathrm{eV}$. Also, Auger electrons are released with energies of $\leqslant 2800 \mathrm{eV}(2800,2300,1000 \mathrm{eV}, \ldots$; see Fig. 7).

The attenuation length of the $8.2-\mathrm{keV}$ photon in tungsten is $4.09 \mu \mathrm{m}$, and thus, within the 200-nm layer the photoelectrons are produced homogeneously. For a simple estimation, let us assume that one sixth of them is traveling in the direction of the diamond substrate. Flying through the media, electrons will lose their energy according to their energy loss function (see Fig. 8). The electron inelastic mean free path and the corresponding energy loss function are calculated with help of the CDF formalism, Eq. (1), where the necessary coefficients were taken from optical data, ${ }^{53,54}$ following the procedure described in Refs. 51,57, and 58. 


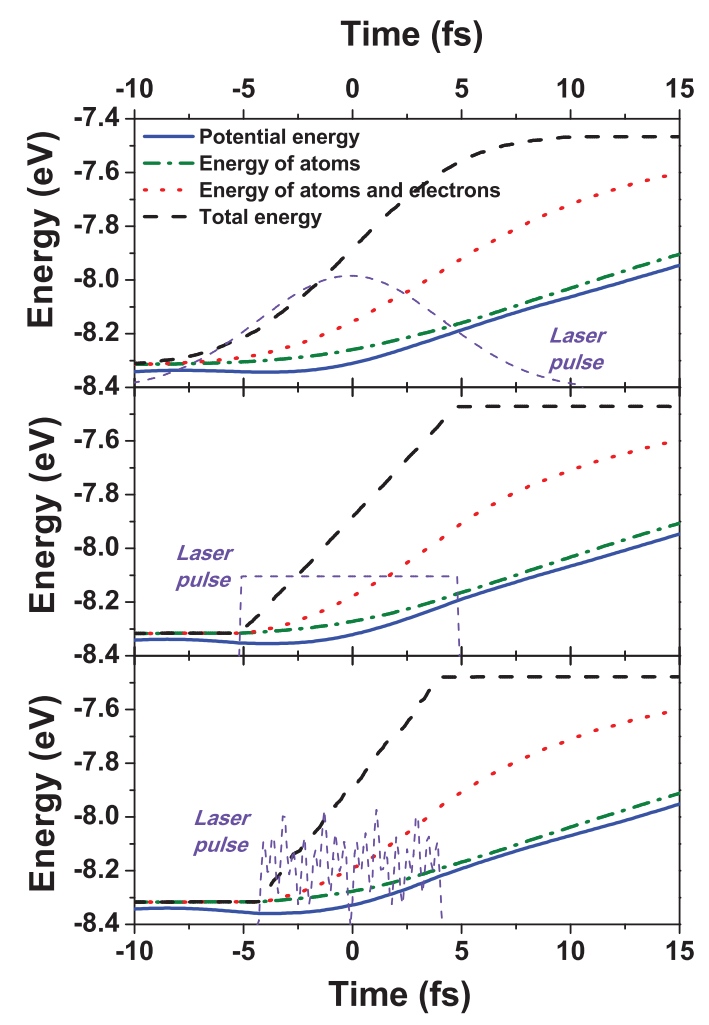

FIG. 6. (Color online) Energy of atoms and electrons in diamond as a function of time, recorded during and after three laser pulses with different pulse envelopes: (top) Gaussian, (middle) flat top, and (bottom) SASE.

Electrons, homogeneously produced in the tungsten layer via photoabsorption, on average have an energy of $2100 \mathrm{eV}$ when reaching diamond. Secondary impact-ionized or Auger electrons with energies below $\sim 3000 \mathrm{eV}$ cannot reach the substrate and deposit all their energy within the tungsten.

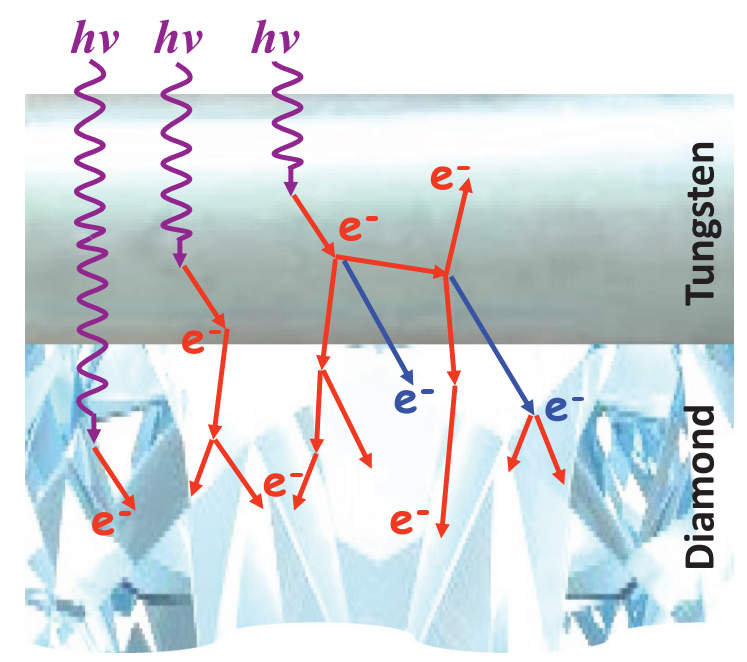

FIG. 7. (Color online) Schematic picture of the multilayer tungsten-diamond structure, irradiated with a laser pulse. The violet lines represent photons $(h v)$, the red arrows stand for photoelectrons and secondary electrons (marked with $e^{-}$), and the blue arrows represent Auger electrons.

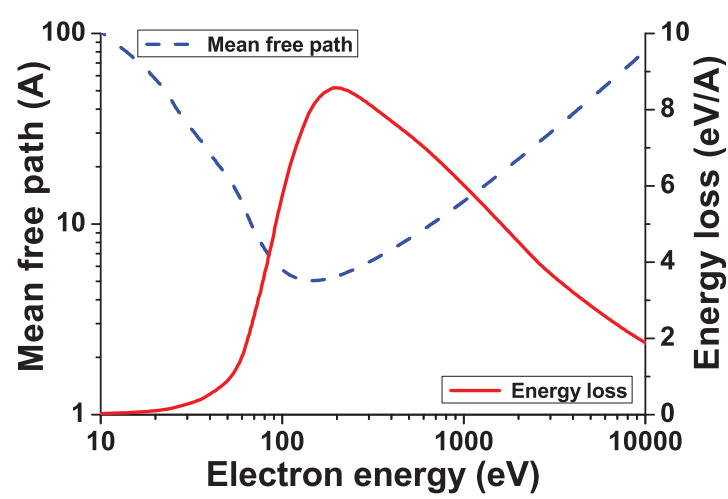

FIG. 8. (Color online) Electron inelastic mean free path and the corresponding energy loss function in tungsten.

Inside the diamond, the electrons from tungsten will further lose their energy into the material on a length scale of $\sim 100 \mathrm{~nm}$.

Table I sums up the energy that electrons from tungsten bring to diamond, giving the values of the total deposited dose for different fluences of the laser pulse presented in Ref. 28. Table I shows that the presence of the 200-nm-thick layer of tungsten induces the shift of the damage threshold. The damage now occurs at a fluence between 59 and 99 $\mathrm{J} / \mathrm{cm}^{2}$, the same as observed in the experiment: ${ }^{28}$ for the higher fluence the damage was reported, while for the lower fluence no damage was observed. The electrons from tungsten dramatically increase the total deposited dose in diamond, overcoming the damage threshold value of the deposited dose per atom, $0.7 \mathrm{eV} /$ atom. This confirms the earlier finding that the presence of a heavier element covering a low- $Z$ material can enhance the damage in the low- $Z$ material. ${ }^{71}$

Despite the fact that part of the energy deposited by radiation in tungsten is transferred by electrons to diamond, the radiation dose remaining in tungsten is still sufficiently high to evaporate the tungsten layer (these doses were estimated to be 2.4, 3.0, and 7.2 eV/atom for the three considered fluences), in agreement with the experimental observation.

The energy brought to the substrate from the top layer depends on the material properties, its thickness, and the photon energy. Thus, a dedicated analysis of the damage threshold is necessary for any specific multilayer composition. ${ }^{71}$

TABLE I. Comparison of photoabsorption in diamond with the energy deposition made by electrons from the 200-nm tungsten layer put on top of diamond. $F$ denotes the fluence of the incoming laser pulse, $D_{\mathrm{ph}}(C)$ is the photoabsorbed dose in diamond, and $D_{e}(C)$ is the dose deposited in diamond by the electrons from tungsten. For comparison, the last column shows the calculated damage threshold in diamond.

\begin{tabular}{lccc}
\hline \hline$F\left(\mathrm{~J} / \mathrm{cm}^{2}\right)$ & $D_{\mathrm{ph}}(C)(\mathrm{eV} /$ atom $)$ & $\begin{array}{c}D_{e}(C) \\
(\mathrm{eV} / \text { atom })\end{array}$ & $\begin{array}{c}\text { Damage threshold } \\
(\mathrm{eV} / \text { atom })\end{array}$ \\
\hline 59 & 0.027 & 0.66 & \\
99 & 0.046 & 1.19 & 0.7 \\
220 & 0.10 & 2.65 & \\
\hline \hline
\end{tabular}




\section{CONCLUSION}

In the present work, we have extended the recently developed hybrid model for the nonthermal phase transition in diamond ${ }^{38}$ to the hard $\mathrm{x}$-ray regime. The combination of different numerical approaches within one model enables us to address in a computationally efficient way the nonequilibrium electron kinetics, secondary cascading due to impact ionizations, Auger decays of $K$-shell holes, atomic dynamics on the evolving many-body potential energy surface, and the modification of the electronic band structure.

Our model was employed to study the graphitization of diamond under femtosecond laser-pulse irradiation. The diamond damage threshold was calculated for a wide range of photon energies from soft to hard $\mathrm{x}$ rays. Together with the predictions for the VUV energy regime obtained in Ref. 43, the damage threshold was found to keep a constant value of $\sim 0.7 \mathrm{eV} /$ atom in terms of the absorbed dose over the entire range of photon energies.

The transient kinetics of electrons and atoms under the irradiation was analyzed. It was shown that for hard x-ray photon energies, the secondary electron cascading and the corresponding transfer of electrons to the conduction band take up to a hundred femtoseconds longer than in the case of the VUV-XUV irradiation. They also affect the atomic dynamics, delaying the phase transition from diamond to graphite. Both effects, the electronic one and the atomic one, could be resolved experimentally.
The analysis of the temporal shape of the laser pulse confirmed that for the femtosecond pulses in the linear optics regime the damage threshold was not affected by the specific shape of the laser pulse (at the fixed pulse duration and fluence). Various temporal shapes of the analyzed pulses, the Gaussian profile, the flat-top profile, and the SASE pulse, resulted in only minor changes in the transient electron kinetics during the pulse.

In the context of the recent work of Ref. 28, we also showed that a layer of high- $Z$ material (tungsten) put on top of diamond can significantly enhance the radiation damage within diamond due to ionizations caused by additional secondary electrons coming from the surface layer. These high-energy electrons, produced by the x-ray pulse within the surface layer, can transfer large amounts of energy into the interior of the sample. The cumulative damage, combining the primary damage in diamond and the damage by photoelectrons from the high- $Z$ surface layer, can then be observed by the significant lowering of the damage threshold fluence below the pure-diamond value.

\section{ACKNOWLEDGMENTS}

The authors thank A. Aquila, J. Gaudin, A. Mancuso, R. Santra, F. Uhlén, and O. Vendrell for inspiring discussions.
*Corresponding author: nikita.medvedev@desy.de

${ }^{1}$ W. Ackermann, G. Asova, V. Ayvazyan, A. Azima, N. Baboi, J. Bähr, V. Balandin, B. Beutner, A. Brandt, A. Bolzmann et al., Nat. Photonics 1, 336 (2007).

${ }^{2}$ P. Emma, R. Akre, J. Arthur, R. Bionta, C. Bostedt, J. Bozek, A. Brachmann, P. Bucksbaum, R. Coffee, F.-J. Decker et al., Nat. Photonics 4, 641 (2010).

${ }^{3}$ D. Pile, Nat. Photonics 5, 456 (2011).

${ }^{4}$ E. Allaria, R. Appio, L. Badano, W. Barletta, S. Bassanese, S. Biedron, A. Borga, E. Busetto, D. Castronovo, P. Cinquegrana et al., Nat. Photonics 6, 699 (2012).

${ }^{5}$ B. Rudek, S.-K. Son, L. Foucar, S. W. Epp, B. Erk, R. Hartmann, M. Adolph, R. Andritschke, A. Aquila, N. Berrah et al., Nat. Photonics 6, 858 (2012).

${ }^{6}$ J. Wark, Nature (London) 466, 35 (2010).

${ }^{7}$ N. Berrah, L. Fang, B. Murphy, T. Osipov, K. Ueda, E. Kukk, R. Feifel, P. van der Meulen, P. Salen, H. T. Schmidt et al., Proc. Natl. Acad. Sci. USA 108, 16912 (2011).

${ }^{8}$ J. Ullrich, A. Rudenko, and R. Moshammer, Annu. Rev. Phys. Chem. 63, 635 (2012).

${ }^{9}$ B. Ziaja, H. Wabnitz, F. Wang, E. Weckert, and T. Möller, Phys. Rev. Lett. 102, 205002 (2009).

${ }^{10}$ B. Ziaja, H. N. Chapman, R. Fäustlin, S. Hau-Riege, Z. Jurek, A. V. Martin, S. Toleikis, F. Wang, E. Weckert, and R. Santra, New J. Phys. 14, 115015 (2012).

${ }^{11}$ N. Medvedev, U. Zastrau, E. Förster, D. O. Gericke, and B. Rethfeld, Phys. Rev. Lett. 107, 165003 (2011).

${ }^{12}$ L. Müller, C. Gutt, B. Pfau, S. Schaffert, J. Geilhufe, F. Büttner, J. Mohanty, S. Flewett, R. Treusch, S. Düsterer et al., Phys. Rev. Lett. 110, 234801 (2013).
${ }^{13}$ M. Beye, F. Sorgenfrei, W. F. Schlotter, W. Wurth, and A. Föhlisch, Proc. Natl. Acad. Sci. USA 107, 16772 (2010).

${ }^{14}$ S. M. Vinko, O. Ciricosta, B. I. Cho, K. Engelhorn, H.-K. Chung, C. R. D. Brown, T. Burian, J. Chalupský, R. W. Falcone, C. Graves et al., Nature (London) 482, 59 (2012).

${ }^{15}$ B. Nagler, U. Zastrau, R. R. Fäustlin, S. M. Vinko, T. Whitcher, A. J. Nelson, R. Sobierajski, J. Krzywinski, J. Chalupsky, E. Abreu et al., Nat. Phys. 5, 693 (2009).

${ }^{16}$ S. M. Vinko, U. Zastrau, S. Mazevet, J. Andreasson, S. Bajt, T. Burian, J. Chalupsky, H. N. Chapman, J. Cihelka, D. Doria et al., Phys. Rev. Lett. 104, 225001 (2010).

${ }^{17}$ O. Renner, P. Sauvan, E. Dalimier, C. Riconda, F. B. Rosmej, S. Weber, P. Nicolai, O. Peyrusse, I. Uschmann, S. Hofer et al., in Spectral Line Shapes: Volume 15-19th International Conference on Spectral Line Shapes, AIP Conf. Proc. No. 1058 (AIP, New York, 2008), pp. 341-348.

${ }^{18}$ U. Zastrau, C. Fortmann, R. Fäustlin, L. Cao, T. Döppner, S. Düsterer, S. Glenzer, G. Gregori, T. Laarmann, H. Lee et al., Phys. Rev. E 78, 066406 (2008).

${ }^{19}$ U. Zastrau, T. Burian, J. Chalupsky, T. Döppner, T. Dzelzainis, R. Fäustlin, C. Fortmann, E. Galtier, S. Glenzer, G. Gregori et al., Laser Part. Beams 30, 45 (2012).

${ }^{20}$ C. E. Graves, A. H. Reid, T. Wang, B. Wu, S. de Jong, K. Vahaplar, I. Radu, D. P. Bernstein, M. Messerschmidt, L. Müller et al., Nat. Mater. 12, 293 (2013).

${ }^{21}$ M. Harmand, R. Coffee, M. Bionta, M. Chollet, D. French, D. M. Zhu, D. T. Fritz, H. Lemke, N. Medvedev, B. Ziaja et al., Nat. Photonics 7, 215 (2013). 
${ }^{22}$ R. Riedel, A. Al-Shemmary, M. Gensch, T. Golz, M. Harmand, N. Medvedev, M. Prandolini, K. Sokolowski-Tinten, S. Toleikis, U. Wegner et al., Nat. Commun. 4, 1731 (2013).

${ }^{23}$ S. P. Hau-Riege and T. Pardini, J. Appl. Phys. 112, 114904 (2012).

${ }^{24}$ A. Barty, S. Boutet, M. J. Bogan, S. Hau-Riege, S. Marchesini, K. Sokolowski-Tinten, N. Stojanovic, R. Tobey, H. Ehrke, A. Cavalleri et al., Nat. Photonics 2, 415 (2008).

${ }^{25}$ F. Wang, E. Weckert, B. Ziaja, D. S. D. Larsson, and D. van der Spoel, Phys. Rev. E 83, 031907 (2011).

${ }^{26}$ I. Grguraš, A. R. Maier, C. Behrens, T. Mazza, T. J. Kelly, P. Radcliffe, S. Düsterer, A. K. Kazansky, N. M. Kabachnik, T. Tschentscher et al., Nat. Photonics 6, 852 (2012).

${ }^{27}$ A. Aquila, C. Ozkan, R. Sobierajski, V. Hájková, T. Burian, J. Chalupsky, L. Juha, M. Störmer, H. Ohashi, T. Koyama et al., in SPIE Optics + Optoelectronics, edited by L. Juha, S. Bajt, R. London, R. Hudec, and L. Pina (International Society for Optics and Photonics, Bellingham, WA, 2013), 87770H.

${ }^{28}$ F. Uhlén, D. Nilsson, A. Holmberg, H. M. Hertz, C. G. Schroer, F. Seiboth, J. Patommel, V. Meier, R. Hoppe, A. Schropp et al., Opt. Express 21, 8051 (2013).

${ }^{29}$ M. Antonelli, M. Di Fraia, A. Tallaire, J. Achard, S. Carrato, R. H. Menk, G. Cautero, D. Giuressi, W. H. Jark, G. Biasiol et al., in SPIE Optical Engineering + Applications, edited by S. P. Moeller, M. Yabashi, and S. P. Hau-Riege (International Society for Optics and Photonics, Bellingham, WA, 2012), 85040D.

${ }^{30}$ M. Antonelli, M. Di Fraia, S. Carrato, G. Cautero, R. H. Menk, W. H. Jark, T. Ganbold, G. Biasiol, C. Callegari, M. Coreno, A. De Sio, and E. Pace, Nucl. Instrum. And Meth. A 730, 164 (2013).

${ }^{31}$ C. David, S. Gorelick, S. Rutishauser, J. Krzywinski, J. VilaComamala, V. A. Guzenko, O. Bunk, E. Färm, M. Ritala, M. Cammarata et al., Sci. Rep. 1, 57 (2011).

${ }^{32}$ P. Lorazo, L. J. Lewis, and M. Meunier, Phys. Rev. B 73, 134108 (2006).

${ }^{33}$ B. Ziaja and N. Medvedev, High Energy Density Phys. 8, 18 (2012).

${ }^{34}$ O. Keski-Rahkonen and M. O. Krause, At. Data Nucl. Data Tables 14, 139 (1974).

${ }^{35}$ N. Medvedev and B. Rethfeld, Europhys. Lett. 88, 55001 (2009).

${ }^{36}$ B. Ziaja, R. A. London, and J. Hajdu, J. Appl. Phys. 97, 064905 (2005).

${ }^{37}$ B. Rethfeld, A. Kaiser, M. Vicanek, and G. Simon, Phys. Rev. B 65, 214303 (2002).

${ }^{38}$ N. Medvedev, H. O. Jeschke, and B. Ziaja, New J. Phys. 15, 015016 (2013).

${ }^{39}$ H. O. Jeschke, M. E. Garcia, and K. H. Bennemann, Phys. Rev. B 60, R3701 (1999).

${ }^{40}$ H. O. Jeschke, M. E. Garcia, and K. H. Bennemann, Phys. Rev. Lett. 87, 015003 (2001).

${ }^{41}$ S. K. Sundaram and E. Mazur, Nat. Mater. 1, 217 (2002).

${ }^{42}$ N. A. Medvedev, H. O. Jeschke, and B. Ziaja, Proc. SPIE 8777, 877709 (2013).

${ }^{43}$ J. Gaudin, N. Medvedev, J. Chalupský, T. Burian, S. DastjaniFarahani, V. Hájková, M. Harmand, H. O. Jeschke, L. Juha, M. Jurek et al., Phys. Rev. B 88, 060101(R) (2013).
${ }^{44}$ D. A. Chapman and D. O. Gericke, Phys. Rev. Lett. 107, 165004 (2011).

${ }^{45}$ R. R. Fäustlin, T. Bornath, T. Döppner, S. Düsterer, E. Förster, C. Fortmann, S. H. Glenzer, S. Göde, G. Gregori, R. Irsig et al., Phys. Rev. Lett. 104, 125002 (2010).

${ }^{46}$ S. P. Hau-Riege, Phys. Rev. E 87, 053102 (2013).

${ }^{47}$ O. Osmani, N. Medvedev, M. Schleberger, and B. Rethfeld, Phys. Rev. B 84, 214105 (2011).

${ }^{48}$ M. C. Ridgway, T. Bierschenk, R. Giulian, B. Afra, M. D. Rodriguez, L. L. Araujo, A. P. Byrne, N. Kirby, O. H. Pakarinen, F. Djurabekova et al., Phys. Rev. Lett. 110, 245502 (2013).

${ }^{49}$ N. A. Medvedev and B. Rethfeld, Proc. SPIE 8077, 80770Q (2011).

${ }^{50}$ N. Medvedev and B. Rethfeld, New J. Phys. 12, 073037 (2010).

${ }^{51}$ A. Akkerman, T. Boutboul, A. Breskin, R. Chechik, A. Gibrekhterman, and Y. Lifshitz, Phys. Status Solidi B 198, 769 (1996).

${ }^{52}$ M. Rabi, Quantum Mechanics in Physics and Chemistry with Applications to Biology (Phi Learning, New Delhi, 2011).

${ }^{53}$ E. D. Palik, Handbook of Optical Constants of Solids, Academic Press Handbook Series Vol. 1 (Academic, Orlando, FL, 1985).

${ }^{54}$ B. Henke, E. Gullikson, and J. Davis, At. Data Nucl. Data Tables 54, 181 (1993).

${ }^{55} \mathrm{~S}$. Adachi, The Handbook on Optical Constants of Semiconductors: In Tables and Figures (World Scientific, Singapore, 2012).

${ }^{56}$ J. Bearden and A. Burr, Rev. Mod. Phys. 39, 125 (1967).

${ }^{57}$ R. H. Ritchie and A. Howie, Philos. Mag. 36, 463 (1977).

${ }^{58}$ N. Medvedev, in International Symposium on High Power Laser Ablation 2012, AIP Conf. Proc. No. 1464 (AIP, New York, 2012), p. 582.

${ }^{59}$ J. Chihara, J. Phys. Condens. Matter 12, 231 (2000).

${ }^{60}$ M. Parrinello and A. Rahman, Phys. Rev. Lett. 45, 1196 (1980).

${ }^{61}$ H. O. Jeschke, Ph.D. thesis, Freie Universität Berlin, 2000.

${ }^{62}$ C. H. Xu, C. Z. Wang, C. T. Chan, and K. M. Ho, J. Phys. Condens. Matter 4, 6047 (1992)

${ }^{63}$ A. T. Collins, Diamond Relat. Mater. 1, 457 (1992).

${ }^{64}$ J. F. Prins, Radiat. Effects Defects Solids 156, 173 (2001).

${ }^{65}$ N. Medvedev, B. Ziaja, M. Cammarata, M. Harmand, and S. Toleikis, Contrib. Plasma Phys. 53, 347 (2013).

${ }^{66}$ C. L. Lu, C. P. Chang, Y. C. Huang, J. H. Ho, C. C. Hwang, and M. F. Lin, J. Phys. Soc. Jpn. 76, 024701 (2007).

${ }^{67}$ K. Yoshizawa, T. Kato, and T. Yamabe, J. Chem. Phys. 105, 2099 (1996).

${ }^{68}$ J. Amann, W. Berg, V. Blank, F.-J. Decker, Y. Ding, P. Emma, Y. Feng, J. Frisch, D. Fritz, J. Hastings et al., Nat. Photonics 6, 693 (2012).

${ }^{69}$ Y. Ding, A. Brachmann, F.-J. Decker, D. Dowell, P. Emma, J. Frisch, S. Gilevich, G. Hays, P. Hering, Z. Huang et al., Phys. Rev. Lett. 102, 254801 (2009).

${ }^{70}$ L. Englert, B. Rethfeld, L. Haag, M. Wollenhaupt, C. SarpeTudoran, and T. Baumert, Opt. Express 15, 17855 (2007).

${ }^{71}$ B. Ziaja, H. N. Chapman, R. Santra, T. Laarmann, E. Weckert, C. Bostedt, and T. Möller, Phys. Rev. A 84, 033201 (2011). 